\title{
UNIVERSAL CONNECTIONS ON LIE GROUPOIDS
}

\author{
EFSTATHIOS VASSILIOU and APOSTOLOS NIKOLOPOULOS
}

\author{
Received 7 November 2001
}

\begin{abstract}
Given a Lie groupoid $\Omega$, we construct a groupoid $J_{1} \Omega$ equipped with a universal connection from which all the connections of $\Omega$ are obtained by certain pullbacks. We show that this general construction leads to universal connections on principal bundles (considered by García (1972)) and universal linear connections on vector bundles (ultimately related with those of Cordero et al. (1989)).
\end{abstract}

2000 Mathematics Subject Classification: 58H05, 53C05, $22 \mathrm{~A} 22$.

1. Introduction. Many problems in differential geometry of fiber bundles and applications lead to the consideration of a whole family of connections instead of an individual one. This is, for example, the case of the Chern-Weil homomorphism, the universal holonomy theory, parametric models in statistical theory, variational problems in classical field theory, and so forth. In such cases, things are simplified by constructing universal connections from which we deduce all the connections considered (for relevant comments and examples, we refer to $[3,4])$.

Universal connections have been studied in, for example, [4, 8, 12, 13], to name but a few of the earlier attempts to the subject. Narasimhan and Ramanan [12], based on the Stiefel bundles and unitary groups, proved the existence of universal bundles for principal bundles with a compact Lie group as a structure group. This result was extended to the case of arbitrary connected Lie groups in [13]. However, [8] has a rather algebraic nature showing that the geometric construction of [12] corresponds to the identity map of the Weil algebra of the Lie algebra of a connected compact Lie group. On the other hand, a universal connection in the sense of [4] is defined by an appropriate 1-form on the 1-jet bundle of sections of a given principal bundle.

Motivated by this approach, we study the existence of universal connections on Lie groupoids. The geometry of Lie groupoids and algebroids is a topic of current research which, beyond its significance per se, has found numerous applications in many areas (see, e.g., [2, 9, 10, 18]). In this framework, universal connections might be an interesting complement of the theory of groupoid connections.

More explicitly, in Theorem 4.5, we prove that, roughly speaking, a given Lie groupoid $\Omega$ determines a Lie groupoid $J_{1} \Omega$ equipped with a universal connection from which all the connections of $\Omega$ are derived (by appropriate pullbacks). 
Here, $J_{1} \Omega$ is the pullback of $\Omega$ via $\pi_{1}: Q_{1} \Omega \rightarrow B$, where $B$ is the base of the groupoid and $Q_{1} \Omega$ is the corresponding connection bundle, whose sections are in bijective correspondence with the connections of $\Omega$.

Before giving the proof of the main result, we study in detail the bundle $Q_{1} \Omega$, the differentiable structure of $J_{1} \Omega$, as well as the Lie algebroid $\mathbf{L}\left(J_{1} \Omega\right)$. We also give an explicit description of certain mappings involved in the construction of the universal connection.

The paper is completed with two applications. The first one explains how García's universal connections on principal bundles fit in this scheme. Namely, in Theorem 5.2, we show that our universal connection induces the one defined in the sense of [4], by passing to the vertex bundle of the Lie groupoid. The second application deals with universal connections for the frame groupoids of vector bundles. In fact, we prove that such connections lead to the existence of universal linear connections for the corresponding vector bundles (Theorem 5.9). As we remark in Scholium 5.10, the previous result can be related with the universal linear connection considered also in [3].

2. Preliminaries. Throughout this paper, all the manifolds and bundles are supposed to be smooth and finite dimensional. For the standard theory of connections on fiber bundles, we refer to [6, 7]. For groupoids and algebroids, we mainly follow the notation and terminology of [9]; however, for the reader's convenience, in this section, we briefly review some facts needed in the sequel.

A groupoid $\Omega$ is a small category all the morphisms of which are invertible. Hence, $\Omega$ is equipped with two projections $\alpha: \Omega \rightarrow B$ and $\beta: \Omega \rightarrow B$ (called source and target, respectively), an inversion $\tau: \Omega \rightarrow \Omega: \xi \mapsto \xi^{-1}$, and a multiplication $\gamma: \Omega * \Omega \rightarrow \Omega:(\eta, \xi) \mapsto \eta \cdot \xi \equiv \eta \xi$, where $\Omega * \Omega$ is the set of the composable morphisms. Here, $B$ is the set of the objects (base of the groupoid). To every element $x \in B$, we assign a corresponding unity (or identity) $\tilde{x}$, the set of unities being naturally identified with $B$ via the object inclusion map $\varepsilon: B \rightarrow \Omega: x \mapsto \tilde{x}$. Consequently, $B$ may be viewed as a subset of the total space $\Omega$.

Some subsets of the groupoid are of special importance for the study of the latter. Namely, we set

$$
\begin{gathered}
\Omega_{x}=\alpha^{-1}(x), \quad \Omega^{y}:=\beta^{-1}(y), \quad \Omega_{x}^{y}:=\alpha^{-1}(x) \cap \beta^{-1}(y), \\
\Omega_{U}:=\alpha^{-1}(U), \quad \Omega^{V}:=\beta^{-1}(V), \quad \Omega_{U}^{V}:=\Omega_{U} \cap \Omega^{V},
\end{gathered}
$$

for every $U, V \subseteq B$ and $x, y \in B$.

As a matter of terminology, $\Omega_{x}$ is the $\alpha$-fiber of $\Omega$ at $x, \Omega^{y}$ is the $\beta$-fiber of $\Omega$ at $y$, and $\Omega_{x}^{x}$ is the isotropy group of $\Omega$ at $x$.

By definition, $\Omega$ is a transitive groupoid if the anchor map

$$
(\beta, \alpha): \Omega \longrightarrow B \times B: \xi \longmapsto(\beta(\xi), \alpha(\xi))
$$


is surjective. Moreover, we speak of a differentiable groupoid if $\Omega$ and $B$ are differentiable manifolds, the mappings $\gamma, \varepsilon$ are differentiable and the projections $\alpha, \beta$ are differentiable submersions. Under these assumptions, the differentiability of the inversion map $\tau$ can also be deduced. The $\alpha$ - and $\beta$-fibers of a differentiable groupoid are regular submanifolds of the total space.

We need the following typical examples of differentiable groupoids:

(a) the trivial groupoid $B \times G \times B$ ( $B$ differentiable manifold and $G$ Lie group) with multiplication given by

$$
(x, g, y) \cdot(y, h, z)=(x, g \cdot h, z)
$$

(b) the frame groupoid $\Pi(E)$ of a vector bundle $E$ defined as follows: if $E_{z}$ denotes the fiber of $E$ over $z \in B$, then

$$
\Pi(E):=\left\{f \in \operatorname{Lis}\left(E_{x}, E_{y}\right) \mid x, y \in B\right\}
$$

and the multiplication is the usual composition of maps. Here, Lis $\left(E_{x}\right.$, $E_{y}$ ) denotes the set of linear isomorphisms of $E_{x}$ onto $E_{y}$;

(c) the inner subgroupoid $G \Omega$ of a given differentiable groupoid $\Omega$, defined by

$$
G \Omega:=\bigcup_{x \in B} \Omega_{x}^{x}
$$

(d) the sets $\Omega_{U}^{U}$, if $U$ is an open subset of $B$ and $\Omega$ is a differential groupoid. A Lie groupoid is a transitive differentiable groupoid. In this case, $(\beta, \alpha)$ is also a submersion (see [9, pages 86-89]) and the isotropy groups are mutually isomorphic Lie groups. We note that every trivial groupoid is Lie, while $\Pi(E)$ (see example (b) above) is a Lie groupoid if the base space $B$ is connected.

Defining morphisms of groupoids in the obvious way (see, e.g., [9, Definitions I.2.1 and III.1.1]), a Lie groupoid satisfies the following (equivalent) conditions: (T.1) there is an open cover $\mathscr{b}$ of $B$ and local trivializing maps (isomorphisms of differentiable groupoids) of the form

$$
\Sigma_{U}: \Omega_{U}^{U} \rightarrow U \times G \times U, \quad U \in \mathscr{C},
$$

where, for the sake of simplicity, we have set $G:=\Omega_{b}^{b}$ for an arbitrary fixed $b \in B$;

(T.2) there are smooth local sections of $\Omega$ with respect to $\mathscr{C}$, that is, $\sigma_{U}: U \rightarrow \Omega$ such that $\beta \circ \sigma_{U}=\mathrm{id} \mid U$ and $\alpha \circ \sigma_{U}=$ constant.

The maps $\Sigma_{U}$ are related with $\sigma_{U}$ by the formula

$$
\Sigma_{U}(\xi)=\left(\beta(\xi), \sigma_{U}(\beta(\xi))^{-1} \cdot \xi \cdot \sigma_{U}(\alpha(\xi)), \alpha(\xi)\right), \quad \xi \in \Omega
$$

For later reference, we note that there is a bijective correspondence between Lie groupoids and principal fiber bundles: given a Lie groupoid $\Omega$, the quadruples $\left(\Omega_{x}, \Omega_{x}^{x}, B, \beta_{x}:=\beta \mid \Omega_{x}\right), x \in B$, are mutually isomorphic principal bundles, 
called vertex bundles. Conversely, a principal bundle $P$ is the vertex bundle of the gauge groupoid $P \times P / G$ (see, e.g., [9, Proposition II. 1.19]).

3. Connections on groupoids. Before defining connections, we need the fundamental notion of a Lie algebroid, generalizing that of a Lie algebra, and originally due to Pradines ([15]).

Given a vector bundle (v.b., for short) $E \equiv(E, p, B)$, we denote by $\mathscr{S}(E)$ the module of its sections.

DEFINITION 3.1. A Lie algebroid is a vector bundle $E$, such that the module $\mathscr{S}(E)$ is equipped with a Lie bracket $[\cdot, \cdot]$ and there exists a v.b.-morphism $q^{E} \equiv q: E \rightarrow T B$, satisfying the following properties:

$$
q \circ[s, t]=[q \circ s, q \circ t], \quad[s, f t]=f \cdot[s, t]+(q \circ s)(f) \cdot t,
$$

for every $s, t \in \mathscr{Y}(E)$ and $f \in C^{\infty}(B)$.

The manifold $B$ is the base of the algebroid and $q$ is its anchor (flèche).

The Lie algebroid of a differentiable groupoid $\Omega$ is defined to be

$$
\mathbf{L} \Omega:=\bigcup_{x \in B} T_{\tilde{x}} \Omega_{x}
$$

its projection $\pi_{\Omega}$ sending every fiber $T_{\tilde{x}} \Omega_{x}$ to the corresponding $x \in B$, whereas the anchor is $q:=T \beta \mid \mathbf{L} \Omega$. The bracket of $\mathbf{L} \Omega$ is induced by an appropriate bracket on the module of $\alpha$-vertical right invariant vector fields of $\Omega$ (see, e.g., [9, Section III.3]).

In fact, $\mathrm{L}$ is a functor with $\mathbf{L} \phi: \mathbf{L} \Omega \rightarrow \mathbf{L} \Omega^{\prime}$ given by

$$
\left.(\mathbf{L} \phi)\right|_{T_{\tilde{x}} \Omega_{x}}:=T_{\tilde{x}}\left(\left.\phi\right|_{\Omega_{x}}\right), \quad x \in B,
$$

for any morphism of differentiable groupoids $\phi: \Omega \rightarrow \Omega^{\prime}$.

For a Lie groupoid, the sequence of vector bundles

$$
0 \longrightarrow \mathbf{L}(G \Omega) \stackrel{i}{\longrightarrow} \mathbf{L} \Omega \stackrel{q}{\rightarrow} T B \longrightarrow 0
$$

is exact, a fact leading to the following basic definition.

DEFINITION 3.2. A connection on a Lie groupoid $\Omega$ is a right section of $q$, that is, a vector bundle morphism $\gamma: T B \rightarrow \mathbf{L} \Omega$ such that $q \circ \gamma=\mathrm{id}_{T B}$.

In a well-established terminology, $\gamma$ is a splitting of (3.4). If the base $B$ is paracompact, as usual, connections do exist. 
For our purposes, we need another approach to the notion of a connection, involving the sections of an appropriate fiber bundle. Namely, following [11, Section III.1], we set

$$
\left.Q_{1} \Omega\right|_{x}:=\left\{j_{x}^{1} s \mid s \text { is a local section of } \Omega: s(x)=\tilde{x}\right\}
$$

for every $x \in B$, and

$$
Q_{1} \Omega:=\left.\bigcup_{x \in B} Q_{1} \Omega\right|_{x}
$$

where $j_{x}^{1} s$ is the 1 -jet of $s$ at $x$. We also define the mapping

$$
\pi_{1}: Q_{1} \Omega \longrightarrow B: j_{x}^{1} s \longmapsto x
$$

Proposition 3.3. The triplet $\left(Q_{1} \Omega, \pi_{1}, B\right)$ is a fiber bundle.

PROoF. We are based on an explicit use of the local charts of $\Omega$ (compare with the proof of [11, Proposition III.1a]).

As in condition (T.2) of Section 2, we may construct smooth local sections $\sigma_{U}: U \rightarrow \Omega$, so that $\mathscr{C}$ is also an atlas of the manifold $B$. For every $U \in \mathscr{C}$, let

$$
\begin{gathered}
\Phi_{U}: U \times \mathbb{B} \longrightarrow T U \quad(\text { where } \mathbb{B} \text { is the model space of } B), \\
\Sigma_{U}: \Omega_{U}^{U} \longrightarrow U \times G \times U \quad\left(G \cong \Omega_{b}^{b}, b \in B\right)
\end{gathered}
$$

be the induced trivializations of $T B$ and $\Omega$, respectively. We also consider the map

$$
k: T U \times T G \times T U \longrightarrow T_{e} G:(u, v, w) \longmapsto T_{g} \rho_{g^{-1}}(v)
$$

if $v \in T_{g} G$ and $\rho_{g}$ denotes the right translation of $G$ by $g$. Taking a fixed linear isomorphism $\ell: T_{e} G \rightarrow \mathbb{G}$, where $\mathbb{G}$ is the model space of $G$ and $e \equiv \tilde{b}$, we can define the smooth map

$$
F_{U}: \pi_{1}^{-1}(U) \longrightarrow U \times L(\mathbb{B}, \mathbb{G}): j_{x}^{1} s \longmapsto\left(x, \ell \circ k \circ T_{x}\left(\Sigma_{U} \circ s\right) \circ\left(\Phi_{U} \mid\{x\} \times \mathbb{B}\right)\right) .
$$

We obtain a chart of $Q_{1} \Omega$ since $F_{U}$ has a smooth inverse. In fact, the latter is given by $(x, f) \mapsto j_{x}^{1} s$, the jet $j_{x}^{1} s$ being determined by the conditions

$$
s(x)=\tilde{x}, \quad T_{x} s=T\left(\Sigma_{U}\right)^{-1} \circ\left[\operatorname{id}_{T U} \times\left(\ell^{-1} \circ f \circ\left(\Phi_{U} \mid\{x\} \times \mathbb{B}\right)^{-1}\right) \times 0\right] .
$$

The compatibility of the previous charts is established by a direct verification; hence, $Q_{1} \Omega$ becomes a differentiable manifold.

Finally, if $\mathrm{pr}_{1}: U \times L(\mathbb{B}, \mathbb{G}) \rightarrow U$ is the projection to the first factor, we see that $\operatorname{pr}_{1} \circ F_{U}$ coincides with $\pi_{1} \mid \pi_{1}^{-1}(U)$, thus concluding the proof. 
Definition 3.4. The fiber bundle $\left(Q_{1} \Omega, \pi_{1}, B\right)$ is called the connection bundle of the groupoid $\Omega$.

The previous terminology is justified by the following proposition.

Proposition 3.5. There is a bijective correspondence between the smooth sections of $Q_{1} \Omega$ and the connections of the Lie groupoid $\Omega$.

Proof. A connection $\gamma$ induces the section $S^{\gamma}: B \rightarrow Q_{1} \Omega$ given by $S^{\gamma}(x):=$ $j_{x}^{1} s$, where $s$ is a local section of $\Omega$ defined on an open neighborhood of $x$, with $s(x)=\tilde{x}$ and $T_{x} s=\gamma \mid T_{x} B$.

Conversely, let $S: B \rightarrow Q_{1} \Omega: x \mapsto j_{x}^{1} s$ be a smooth section. Then, the map $\gamma^{S}: T B \rightarrow \mathbf{L} \Omega$, with $\gamma^{S} \mid T_{x} B:=T_{x} S$, is a connection on $\Omega$.

It is easily checked that the assignments $\gamma \mapsto S^{\gamma}$ and $S \mapsto \gamma^{S}$ are mutually inverse.

4. Universal connections. We come now to the main part of this paper, treating the construction of universal connections for Lie groupoids. This means, roughly speaking, that we look for a connection (on an appropriate groupoid) from which we can derive all the connections of a given groupoid.

Let $\Omega$ be a Lie groupoid. Using the connection bundle $\left(Q_{1} \Omega, \pi_{1}, B\right)$ (see Proposition 3.3 and Definition 3.4), we give the following definition.

DEFINITION 4.1. The 1-jet groupoid of $\Omega$, denoted by $J_{1} \Omega$, is the pullback groupoid of $\Omega$ by $\pi_{1}$, that is, $J_{1} \Omega:=\pi_{1}^{*} \Omega$.

We recall that the total space $J_{1} \Omega \subseteq Q_{1} \Omega \times \Omega \times Q_{1} \Omega$ is the set

$$
\left\{\left(j_{y}^{1} s, \xi, j_{x}^{1} t\right) \mid s \in \pi_{1}^{-1}(y), t \in \pi_{1}^{-1}(x), \xi \in \Omega_{x}^{y} ; x, y \in B\right\}
$$

and the operations of $J_{1} \Omega$ are defined similarly to those of a trivial groupoid (see example (a) of Section 2 and [9, Definition I.2.11]). The projections, the inversion, the identity map, and the multiplication of $J_{1} \Omega$ are denoted by $a_{1}$, $\beta_{1}, \tau_{1}, \varepsilon_{1}$, and $\gamma_{1}$, respectively.

We note that $J_{1} \Omega$ here is not the first prolongation groupoid, often denoted also by the same symbol.

Proposition 4.2. The 1-jet groupoid $J_{1} \Omega$ is a Lie groupoid.

PROoF. The groupoid $J_{1} \Omega$ is differentiable, as a regular submanifold of the trivial groupoid $Q_{1} \Omega \times \Omega \times Q_{1} \Omega$. Indeed, using the notations included in the proof of Proposition 3.3, the differential structure is determined by the charts of the form

$$
\Psi_{U}: J_{1} \Omega \cap\left(\pi_{1}^{-1}(U) \times \Omega \times \pi_{1}^{-1}(U)\right) \stackrel{\simeq}{\longrightarrow} U \times L(\mathbb{B}, \mathbb{G}) \times G \times U \times L(\mathbb{B}, \mathbb{G}),
$$


given by

$$
\Psi_{U}\left(j_{x}^{1} s, \xi, j_{y}^{1} t\right)=\left(F_{U}\left(j_{x}^{1} s\right), p_{2}\left(\Sigma_{U}(\xi)\right), F_{U}\left(j_{y}^{1} t\right)\right)
$$

with $U$ running the domains of the local sections of $\Omega$. The inverse of $\Psi_{U}$ is

$$
\Psi_{U}^{-1}(x, f, g, y, h)=\left(F_{U}^{-1}(x, f), \Sigma_{U}^{-1}(x, g, y), F_{U}^{-1}(y, h)\right) .
$$

On the other hand, the local expressions of $\alpha_{1}, \beta_{1}$, and $\left(\beta_{1}, \alpha_{1}\right)$, relative to the previous chart are given, respectively, by the projections $\mathrm{pr}_{3}, \mathrm{pr}_{1}$, and $\left(\mathrm{pr}_{1}, \mathrm{pr}_{3}\right)$ of the following 3-factor product:

$$
(U \times L(\mathbb{B}, \mathbb{G})) \times G \times(U \times L(\mathbb{B}, \mathbb{G})) ;
$$

thus, the former maps are (surjective) submersions. Finally, $\gamma_{1}$ and $\varepsilon_{1}$ are smooth since their local expressions are, respectively,

$$
\begin{gathered}
\left(\left(x, f, g_{1}, y, h\right),\left(y, h, g_{2}, z, k\right)\right) \longmapsto\left(x, f, g_{1} \cdot g_{2}, z, k\right), \\
(y, h) \longmapsto\left(y, h, 1_{G}, y, h\right),
\end{gathered}
$$

for every $(x, f),(y, h),(z, k) \in U \times L(\mathbb{B}, \mathbb{G})$ and $g_{1}, g_{2} \in G$.

NOTE 4.3. In the previous proof, we have used an explicit form of local charts which will be also needed later.

We will show that the desired universal connection is one defined on $J_{1} \Omega$. Before its explicit description, we need a few preliminary facts. First, as an immediate consequence of the relative definitions, we obtain that (see also [5, Section 1])

$$
\mathbf{L}\left(J_{1} \Omega\right)=\left\{(u, v): T \pi_{1}(u)=T \beta(v)\right\} \subseteq T\left(Q_{1} \Omega\right) \times \mathbf{L} \Omega .
$$

Here, $(u, v) \equiv(u, v, 0)$, the vector $0 \in T\left(Q_{1} \Omega\right)$ being omitted for the sake of convenience. Hence,

$$
\mathbf{L}\left(J_{1} \Omega\right) \cong T\left(Q_{1} \Omega\right) \times_{T B} \mathbf{L} \Omega .
$$

Moreover, $\mathbf{L}\left(J_{1} \Omega\right)$ is a Lie algebroid over $Q_{1} \Omega$ with anchor (the restriction of) the projection to the first factor $P_{1}: \mathbf{L}\left(J_{1} \Omega\right) \rightarrow T\left(Q_{1} \Omega\right)$. On the other hand, the restriction of the second projection $P_{2}: \mathbf{L}\left(J_{1} \Omega\right) \rightarrow \mathbf{L} \Omega$ is a v.b.-morphism covering $\pi_{1}: Q_{1} \Omega \rightarrow B$.

We consider now the evaluation map

$$
\mathrm{Ev}: \pi_{1}^{*}(T B)=Q_{1} \Omega \times_{B} T B \longrightarrow \mathbf{L} \Omega:\left(j_{x}^{1} s, v\right) \longmapsto T_{x} s(v), \quad v \in T_{x} B
$$


LEMMA 4.4. The evaluation map $\mathrm{Ev}$ is differentiable.

Proof. The charts $F_{U}$ and $\Phi_{U}$ of the bundles $Q_{1} \Omega$ and $T B$, respectively, induce the corresponding chart $\left(\pi_{1}^{-1}(U) \times_{U} T U, \Psi_{U}^{1}\right)$ of $Q_{1} \Omega \times_{B} T B$, with

$$
\Psi_{U}^{1}\left(j_{x}^{1} s, v\right):=\left(x, D \bar{s}(\phi(x)),\left(p_{2} \circ \Phi_{U}^{-1}\right)(v)\right) \in U \times L(\mathbb{B}, \mathbb{G}) \times \mathbb{B},
$$

where $\bar{s}$ is the local expression of $s$ and $(U, \phi)$ is the chart of $B$ inducing the corresponding v.b.-chart $\Phi_{U}$ (see the proof of Proposition 3.3).

Applying now the Lie functor $\mathbf{L}$ on $\Sigma_{U}$, after the identification $\Phi_{U}: U \times B \stackrel{\simeq}{\rightarrow}$ $T U$, we obtain the diffeomorphism $\psi_{U}:\left.\mathbf{L} \Omega\right|_{U} \rightarrow U \times \mathbb{B} \times \mathbb{G}$. The local expression of Ev, relative to $\Psi_{U}^{1}$ and $\psi_{U}$, is

$$
\mathrm{Ev}_{\mathrm{loc}}: U \times L(\mathbb{B}, \mathbb{G}) \times \mathbb{B} \longrightarrow U \times \mathbb{B} \times \mathbb{G}:(x, f, m) \longmapsto(x, m, \mathrm{ev}(f, m)),
$$

where ev $: L(\mathbb{B}, \mathbb{G}) \times \mathbb{B} \rightarrow \mathbb{G}$ is now the ordinary evaluation map. This proves the desired differentiability.

Based on Proposition 3.5 and denoting by $\pi_{Q}: T\left(Q_{1} \Omega\right) \rightarrow Q_{1} \Omega$ the standard projection of the tangent bundle of $Q_{1} \Omega$, we are in a position to prove the main result of the paper; namely, the following theorem.

THEOREM 4.5 (existence of universal groupoid connections). Let $\Omega$ be a Lie groupoid. Then, the mapping

$$
\bar{\Gamma} \equiv \bar{\Gamma}_{\Omega}: T\left(Q_{1} \Omega\right) \longrightarrow \mathbf{L}\left(J_{1} \Omega\right): u \longmapsto\left(u, \operatorname{Ev}\left(\pi_{Q}(u), T \pi_{1}(u)\right)\right)
$$

is a connection on $J_{1} \Omega$ with the following universal property: if $\Gamma:=P_{2} \circ \bar{\Gamma}$, then the equality

$$
\gamma^{S}=\Gamma \circ T S
$$

holds for every connection $S$ on $\Omega$. Moreover, $\bar{\Gamma}$ is the unique connection satisfying this property.

Proof. First, we see that $\bar{\Gamma}$ is well defined, since $\left(\pi_{Q}(u), T \pi_{1}(u)\right) \in Q_{1} \Omega \times_{B}$ $T B$; thus, we can apply Ev on such pairs. Also, $\bar{\Gamma}$ takes values in $\mathbf{L}\left(J_{1} \Omega\right)$, since $\bar{\Gamma}(u) \in T\left(Q_{1} \Omega\right) \times_{T B} \mathbf{L} \Omega \cong \mathbf{L}\left(J_{1} \Omega\right)$, by virtue of (4.8).

The mapping $\bar{\Gamma}$ is differentiable (by Lemma 4.4 ), as well as a v.b-morphism. Moreover (see also (4.8)), the projection $P_{1}: \mathbf{L}\left(J_{1} \Omega\right) \rightarrow T\left(Q_{1} \Omega\right)$ satisfies the equality $P_{1} \circ \bar{\Gamma}=$ id. Since $P_{1}$ coincides now with the anchor of $\mathbf{L}\left(J_{1} \Omega\right), \bar{\Gamma}$ is a connection of $J_{1} \Omega$ (see Definition 3.2). 
On the other hand, since $T_{y} S(v) \in T_{S(y)} Q_{1} \Omega$, for every $v \in T_{y} B$ and $y \in B$, Proposition 3.5 implies that

$$
\begin{aligned}
(\Gamma \circ T S)(v) & =\left(P_{2} \circ \bar{\Gamma} \circ T S\right)(v) \\
& =\operatorname{Ev}\left(\left(\pi_{Q} \circ T S\right)(v), T\left(\pi_{1} \circ S\right)(v)\right) \\
& =\operatorname{Ev}(S(y), v) \\
& =\gamma^{S}(v),
\end{aligned}
$$

for every $v \in T B$; thus, proving the universal property.

Finally, let $\bar{\Gamma}^{\prime}$ be another connection of $J_{1} \Omega$ satisfying the analog of (4.13) and $\Gamma^{\prime}=P_{2} \circ \bar{\Gamma}^{\prime}$. Then, for any $w \in T\left(Q_{1} \Omega\right)$, there is a local section $S$ of $Q_{1} \Omega$ and a vector $v \in T B$ such that $T S(v)=w$. Therefore, $\Gamma^{\prime}(w)=\gamma^{S}(v)=\Gamma(w)$, which proves the uniqueness property.

REMARKS 4.6. (1) For any morphism $f: \mathrm{L} \Omega_{1} \rightarrow A$ of vector bundles and any morphism of differentiable groupoids $h: \Omega_{2} \rightarrow \Omega_{1}$, we set $h^{*} f:=f \circ \mathbf{L} h$. Since, within an isomorphism, $T S=\mathbf{L}(S \times S)$ (see, e.g., [1, Section 4] and [5, Section $1])$, (4.13) can be reformulated as

$$
\gamma^{S}=(S \times S)^{*} \Gamma .
$$

(2) By virtue of the properties stated in Theorem 4.5, we can legitimately call $\bar{\Gamma} \equiv \bar{\Gamma}_{\Omega}$ the universal connection of $\Omega$.

\section{Applications}

5.1. Universal connections on principal bundles. Our present aim is to relate universal connections on groupoids with their counterparts defined by García (see [4]) on principal bundles. In fact, we show that $\bar{\Gamma}$ produces the structural infinitesimal connection (form) of the vertex bundle.

First, we note that every connection $\gamma$ on the Lie groupoid $\Omega$ is equivalent to a v.b.-morphism $c: \mathbf{L} \Omega \rightarrow \mathbf{L}(G \Omega)$, such that

$$
\gamma \circ q+i \circ c=\mathrm{id}
$$

In order to define connection forms, we consider the (principal) vertex bundle $\Omega_{b} \equiv\left(\Omega_{b}, \Omega_{b}^{b}, B, \beta \mid \Omega_{b}\right)$ for any fixed $b \in B$ (see Section 2). Also, we need the morphism of Lie groupoids $\mathrm{Ad}: \Omega \rightarrow \Pi(\mathbf{L}(G \Omega)$ ) (often called the adjoint representation of $\Omega$ ), defined by $\operatorname{Ad}(\xi)=T L \xi \circ T R_{\xi}^{-1}$, as well as the v.b.-morphism

$$
\mathfrak{R}: T^{\alpha} \Omega \longrightarrow \mathbf{L} \Omega: v \longmapsto T_{\xi} R_{\xi}^{-1}(v), \quad v \in T_{\xi} \Omega_{\alpha \xi}
$$

(see also [9, Proposition III.3.3]). 
LEMMA 5.1. Let $\gamma$ be a connection on $\Omega$. Then, the form $\omega^{\gamma} \in \Lambda^{1}\left(\Omega_{b}, T_{\tilde{b}} \Omega_{b}^{b}\right)$, given by

$$
\omega_{\xi}^{\gamma}(v)=\left[\operatorname{Ad}\left(\xi^{-1}\right) \circ c \circ R\right](v), \quad \xi \in \Omega_{b}, v \in T_{\xi} \Omega_{b},
$$

is a connection form on the vertex bundle $\Omega_{b}$.

Proof. By definition,

$$
\omega_{\xi}^{\gamma}=\left(T_{\xi} L_{\xi}^{-1} \mid T_{\xi}^{\alpha} \Omega\right) \circ\left(T_{\widetilde{\beta \xi}} R_{\xi} \mid \mathbf{L}(G \Omega)\right) \circ\left(c \mid(\mathbf{L} \Omega)_{\beta \xi}\right) \circ T_{\xi} R_{\xi}^{-1}
$$

thus, $\omega_{\xi}^{\gamma} \in L\left(T_{\xi} \Omega_{b}, T_{\tilde{b}} \Omega_{b}^{b}\right)$ for every $\xi \in \Omega_{b}$. Since $\mathfrak{R}$ and Ad are differentiable, $\omega^{\gamma}$ is a differentiable 1-form.

Now, for any $X \in \mathscr{S}\left(T\left(\Omega_{b}^{b}\right)\right)$, we see that $c\left([\operatorname{Ad}(\xi)]\left(X_{\tilde{b}}\right)\right)=[\operatorname{Ad}(\xi)]\left(X_{\tilde{b}}\right)$. Consequently, if $X^{*}$ is the Killing vector field corresponding to $X$,

$$
\begin{aligned}
\omega_{\xi}^{\gamma}\left(X_{b}^{*}\right) & =\omega_{\xi}^{\gamma}\left(T_{\tilde{b}} L_{\xi}\left(X_{\tilde{b}}\right)\right)=\left[\operatorname{Ad}\left(\xi^{-1}\right) \circ c \circ T_{\xi} R_{\xi}^{-1} \circ T_{\tilde{b}} L \xi\right]\left(X_{b}\right) \\
& =\left[\operatorname{Ad}\left(\xi^{-1}\right) \circ \mathcal{C} \circ \operatorname{Ad}(\xi)\right]\left(X_{\tilde{b}}\right)=\left[\operatorname{Ad}\left(\xi^{-1}\right) \circ \operatorname{Ad}(\xi)\right]\left(X_{\tilde{b}}\right) \\
& =X_{\tilde{b}} \equiv X .
\end{aligned}
$$

On the other hand, for every $(v, g) \in T_{\xi} \Omega_{b} \times \Omega_{b}^{b}$,

$$
\begin{aligned}
R_{g}^{*} \omega^{\gamma}(v) & =\omega_{\xi g}^{\gamma}\left(T_{\xi} R_{g}(v)\right)=\left[\operatorname{Ad}(\xi g)^{-1} \circ c \circ T_{\xi g} R_{(\xi g)}^{-1} \circ T_{\xi} R_{g}\right](v) \\
& =\left[\operatorname{Ad}\left(g^{-1}\right) \circ \operatorname{Ad}\left(\xi^{-1}\right) \circ c \circ T_{\xi} R_{\xi}^{-1}\right](v)=\left[\operatorname{Ad}\left(g^{-1}\right)\right]\left(\omega^{\gamma}(v)\right)
\end{aligned}
$$

Equalities (5.5) and (5.6) prove that $\omega^{\gamma}$ is indeed a connection form.

In order to apply the preceding lemma to the case of the universal connection $\bar{\Gamma} \equiv \Gamma$, we need the analytic expression of its splitting $C: \mathbf{L}\left(J_{1} \Omega\right) \rightarrow \mathbf{L}\left(G\left(J_{1} \Omega\right)\right)$. As a matter of fact, for every $\left.v \in \mathbf{L}\left(J_{1} \Omega\right)\right|_{Y}$, with $Y:=\left.j_{y}^{1} s \in\left(Q_{1} \Omega\right)\right|_{y}=\pi_{1}^{-1}(y)$ and $y \in B$, we see that

$$
v=\left(v_{1}, v_{2}, 0_{Y}\right) \in T_{Y}\left(Q_{1} \Omega\right) \times\left.\mathbf{L} \Omega\right|_{y} \times T_{Y}\left(Q_{1} \Omega\right),
$$

with $T \pi_{1}\left(v_{1}\right)=T \beta\left(v_{2}\right)$. Hence, using the analog of (5.1) and the definition of $\bar{\Gamma}$

$$
C(v)=\left(0_{Y}, v_{2}-T_{Y}\left(s \circ \pi_{1}\right)\left(v_{1}\right), 0_{Y}\right),
$$

where $0_{Y}$ denotes the zero element of $T_{Y}\left(Q_{1} \Omega\right)$. As a consequence, we obtain the following theorem. 
THEOREM 5.2. The connection form $\omega^{\Gamma}$ on the vertex bundle of $J_{1} \Omega$, induced by the groupoid universal connection $\Gamma \equiv \bar{\Gamma}$ (see Lemma 5.1), coincides-within an isomorphism - with the universal connection (in the sense of [4]) of the vertex bundle $\Omega_{b}$.

Proof. We fix a $\left.Z \in Q_{1} \Omega\right|_{b}$ and the vertex bundle $\left(\left(J_{1} \Omega\right)_{Z},\left(J_{1} \Omega\right)_{Z}^{Z}, Q_{1} \Omega, p_{1}\right)$ of $J_{1} \Omega$. Then, by virtue of Lemma $5.1, \omega^{\Gamma}$ may be regarded as a connection on the principal bundle $\left(J_{1} \Omega\right)_{Z}$.

Every $v \in T_{F}\left(J_{1} \Omega\right)_{Z}$, where $F=(X, \xi, Z) \in\left(J_{1} \Omega\right)_{Z}$, is identified with a triple of the form $v \equiv\left(v_{1}, v_{2}, 0_{Z}\right)$, with $0_{Z} \in T_{Z}\left(Q_{1} \Omega\right), v_{1} \in T_{X}\left(Q_{1} \Omega\right)$, and $v_{2} \in T_{\xi} \Omega_{b}$. If we express the maps $T_{F} R_{F}^{-1}$ and $\operatorname{Ad}\left(F^{-1}\right)$ in these terms, then, for $X:=j_{\beta \xi}^{1} S$, Lemma 5.1 and (5.8) imply that

$$
\omega_{F}^{\Gamma}(v)=\left(0_{X}, \operatorname{Ad}\left(\xi^{-1}\right)\left(\mathfrak{R}\left(v_{2}\right)-T_{X}\left(s \circ \pi_{1}\right)\left(v_{1}\right)\right), 0_{X}\right)
$$

Also, we consider the 1-jet principal bundle $\left(J^{1}\left(\Omega_{b}\right), \Omega_{b}^{b}, J^{1}\left(\Omega_{b}\right) / \Omega_{b}^{b}, \pi_{1}\right)$ corresponding to the principal bundle $\Omega_{b}$ (see [4, Section 2]), as well as the isomorphism $\left(I_{1}, I_{2}, I_{3}\right)$ between the bundles $\left(J_{1} \Omega\right)_{Z}$ and $J^{1}\left(\Omega_{b}\right)$, determined by

$$
I_{1}:\left(J_{1} \Omega\right)_{Z} \longrightarrow J^{1}\left(\Omega_{b}\right):\left(j_{x}^{1} s, \xi, Z\right) \longmapsto j_{x}^{1}(s \xi)
$$

with $(s \xi)(y):=s(y) \cdot \xi$, for every $y \in \operatorname{dom}(s)$,

$$
\begin{gathered}
I_{2}:\left(J_{1} \Omega\right)_{Z}^{Z} \longrightarrow \Omega_{b}^{b}:(Z, g, Z) \longmapsto g, \\
I_{3}: Q_{1} \Omega \longrightarrow J^{1}\left(\Omega_{b}\right) / \Omega_{b}^{b}: j_{x}^{1} s \longmapsto\left[j_{x}^{1}(s \eta)\right]
\end{gathered}
$$

for an arbitrary $\eta \in \Omega_{b}^{x}$.

Now, $\omega^{\Gamma}$ induces a differentiable 1 -form $\bar{\theta}$ given by

$$
\bar{\theta}_{K}(w):=\left(T I_{2} \circ \omega_{(X, \xi, Y)}^{\Gamma} \circ T I_{1}^{-1}\right)(w),
$$

where $K:=I_{1}(X, \xi, Z)$ and $w \in T_{K}\left(J^{1}\left(\Omega_{b}\right)\right)$. Hence, comparing (5.9) and (5.12) with the definition of the universal connection $\theta$ for ordinary principal bundles (see [4] along with [17]), we conclude that

$$
\bar{\theta}_{K}(w)=\left(T_{\xi} L_{\xi}^{-1} \circ \theta_{K}\right)(w),
$$

which completes the proof.

SchоцIUm 5.3. Since every principal fiber bundle is the vertex bundle of its gauge groupoid, the previous theorem shows that universal connections on principal bundles (in the sense of [4]) can be derived from the general construction given in Theorem 4.5. 
5.2. Universal linear connections. In this section, we prove that the universal connection of the frame groupoid $\Pi(E)$ of a vector bundle $E \equiv(E, \pi, B)$ with connected base $B$ (see example (b) in Section 2 ) induces a universal linear connection on $E$.

First, we need the following general lemma.

LEMMA 5.4. Let $f: V \rightarrow B$ be a differentiable map and $f^{*} E$ be the pullback vector bundle of $E$ by $f$. Then,

$$
f^{*} \Pi(E) \cong \Pi\left(f^{*} E\right)
$$

Proof. The groupoid $f^{*} \Pi(E)$ consists of triples of the form $(y, g, x)$ for all $x, y \in V$ and $g \in \operatorname{Lis}\left(E_{f(x)}, E_{f(y)}\right)$. On the other hand, $\Pi\left(f^{*} E\right)$ consists of pairs $\left(c_{x}^{y}, g\right)$, for all $x, y \in V$ and $g \in \operatorname{Lis}\left(E_{f(x)}, E_{f(y)}\right)$, where $c_{x}^{y}$ denotes the mapping $\{x\} \rightarrow\{y\}$. We get an isomorphism, say $h$, by setting $h(y, g, x)=\left(c_{x}^{y}, g\right)$.

Applying the previous result to the case of $\pi_{1}$, Definition 4.1 implies also the following corollary.

COROLLARY 5.5. The following identification holds true:

$$
J_{1}(\Pi(E))=\pi_{1}^{*}(\Pi(E)) \cong \Pi\left(\pi_{1}^{*} E\right)
$$

It is already known (see, e.g., [11, Section III.2]) that there is a bijective correspondence between the linear connections of the vector bundle $E$ and the connections of $\Pi(E)$. This leads to the following basic proposition.

Proposition 5.6. The universal connection $\Gamma \equiv \bar{\Gamma}_{\Pi(E)}$ of the Lie groupoid $\Pi(E)$ induces a linear connection $C^{\Gamma}$ on the vector bundle $\pi_{1}^{*} E$.

Proof. By its construction, $\Gamma$ is defined on the 1-jet groupoid $J_{1}(\Pi(E))$. Thus, by virtue of Corollary 5.5 , we obtain a connection on $\Pi\left(\pi_{1}^{*} E\right)$ and, in turn, a linear connection $C^{\Gamma}$ on $\pi_{1}^{*} E$.

In order to complete the previous statement, by showing that $C^{\Gamma}$ has indeed a universal property, we find its analytic expression. This is a consequence of the next result, relating linear connections with connections on frame groupoids by means of a simple formula.

To this end, observe that, since $\Pi(E)$ projects to $B$ via the source map $\alpha_{\Pi(E)}$, we may consider the corresponding fiber product $\Pi(E) \times_{B} E$ (with respect to $\left.\alpha_{\Pi(E)}\right)$, the evaluation map

$$
\overline{\mathrm{ev}}: \Pi(E) \times_{B} E \rightarrow E:(f, e) \longmapsto f(e),
$$

and the partial mappings $\overline{\mathrm{ev}}_{e}:\left.\Pi(E)\right|_{\pi(e)} \rightarrow E$, for every $e \in E$. Therefore, we have the following lemma. 
LEMMA 5.7. Let $\gamma: T B \rightarrow \mathbf{L}(\Pi(E))$ be a connection on the frame groupoid $\Pi(E)$. Then, the corresponding linear connection

$$
c^{\gamma}: \pi^{*}(T B)=E \times_{B} T B \longrightarrow T E
$$

on $E$ is given by

$$
c^{\gamma}(e, v):=T_{\mathrm{id}} \overline{\mathrm{ev}}_{e}(\gamma(v)), \quad(e, v) \in \pi^{*}(T B),
$$

where id is the identity map on the fiber $E_{x}$, if $x=\pi(e)$.

Proof. By Proposition 3.5, $\gamma$ induces the section $S^{\gamma}: B \rightarrow Q_{1}(\Pi(E))$ with $S^{\gamma}(x)=j_{x}^{1} s$, where $T_{x} s=\gamma \mid T_{x} B$ and $s(x)=$ id. Now, proceeding as in [11, page 190], $S^{\gamma}$ gives rise to the section

$$
f_{S}: E \longrightarrow J^{1} E: e \longmapsto j_{\pi(e)}^{1}(s \cdot \hat{e})
$$

of the canonical morphism $p: J^{1} E \rightarrow E: j_{z}^{1} t \mapsto t(z)$. Here, $s \cdot \hat{e}$ is the section of $E$, given by

$$
(s \cdot \hat{e})(y)=\overline{\mathrm{ev}}(s(y), e) \in E_{y}
$$

for every $y \in B$. Recall that, by the definition of $\Pi(E), s(y) \in \operatorname{Lis}\left(E_{x}, E_{y}\right)$ and $s(x)$ is the identity map on $E_{x}$.

Finally, based on the relation between linear connections and sections of the canonical morphism $p$ (see, for instance, $[4,16]$ ), we check that

$$
\begin{aligned}
c^{\gamma}(e, v) & =T_{x}(s \cdot \hat{e})(v)=T_{x}\left(\overline{\mathrm{ev}}\left(s, c_{e}\right)\right)(v) \\
& =T_{x}\left(\overline{\mathrm{ev}}_{e} \circ s\right)(v)=T_{\mathrm{id}} \overline{\operatorname{ev}}_{e}\left(T_{x} s(v)\right) \\
& =T_{\mathrm{id}} \overline{\mathrm{ev}}_{e}(\gamma(v)),
\end{aligned}
$$

(where $c_{e}$ is constant) by which we conclude the proof.

To obtain the analog of the previous result for the connections $\Gamma$ and $C^{\Gamma}$, we consider the entities $\pi_{1}^{*} E, Q_{1}(\Pi(E)) \equiv Q_{1}, \bar{\Gamma} \equiv \Gamma$, and $\operatorname{pr}_{1}: \pi_{1}^{*} E \rightarrow Q_{1}$ in place of $E, B, \gamma$, and $\pi$, respectively. Also, analogously to $\overline{\mathrm{ev}}$, we define the map

$$
\overline{\overline{\mathrm{ev}}}: \Pi\left(\pi_{1}^{*} E\right) \times_{Q_{1}} \pi_{1}^{*} E \longrightarrow \pi_{1}^{*} E:(g, m) \longmapsto g(m) .
$$

Proposition 5.8. The linear connection $C^{\Gamma}: \operatorname{pr}_{1}^{*}\left(T Q_{1}\right) \rightarrow T\left(\pi_{1}^{*} E\right)$ is given by

$$
C^{\Gamma}(a, w)=\left(w, T_{\mathrm{id}} \overline{\mathrm{ev}}_{e}\left(T_{x} s\left(T_{X} \pi_{1}(w)\right)\right)\right),
$$

where $a=(X, e) \in \pi_{1}^{*} E \subseteq Q_{1} \times E, X=j_{x}^{1} s$, and $w \in T_{X} Q_{1}$. 
Proof. We outline the main steps of the procedure, omitting the computational details whose verification is a matter of routine.

First, we observe that every $h \in \Pi\left(\pi_{1}^{*} E\right)$ can be written, by the identification of Corollary 5.5, in the form $h=(Y, \bar{h}, X)$ with $X=j_{x}^{1} s, Y=j_{y}^{1} s^{\prime}$, and $\bar{h} \in$ $(\Pi(E))_{x}^{y}$. In these terms, the map $\overline{\overline{\mathrm{eV}}}$ is equivalently given by

$$
\overline{\overline{\mathrm{ev}}}((Y, \bar{h}, X),(X, e))=(Y, \bar{h}(e)) .
$$

Using the projections $p_{1}: J_{1}(\Pi(E)) \rightarrow Q_{1}(\Pi(E))$ and $p_{2}: J_{1}(\Pi(E)) \rightarrow \Pi(E)$, the previous equality becomes

$$
\overline{\overline{\mathrm{ev}}}_{a}=\left(p_{1}, \overline{\mathrm{ev}}_{e} \circ p_{2}\right), \quad a=(X, e),
$$

from which we obtain

$$
T_{\mathrm{id}} \overline{\overline{\mathrm{ev}}}_{a}=\left(\operatorname{Pr}_{1}, T_{\mathrm{id}} \overline{\mathrm{ev}}_{e} \circ \operatorname{Pr}_{2}\right),
$$

where $\operatorname{Pr}_{1}$ and $\operatorname{Pr}_{2}$ are the corresponding differentials of $p_{1}$ and $p_{2}$, respectively, whereas id denotes the identity between appropriate spaces.

On the other hand, by the definition of $\bar{\Gamma}$ and Lemma 5.7 , we conclude that

$$
C^{\Gamma}(a, w)=T_{\mathrm{id}} \overline{\overline{\mathrm{ev}}}_{a}(\bar{\Gamma}(w))=T_{\mathrm{id}} \overline{\overline{\mathrm{ev}}}_{a}\left(w, T_{X} s\left(T_{X} \pi_{1}(w)\right)\right) .
$$

The desired expression is now an immediate consequence of (5.26) and (5.27).

For a given section $S$ of $Q_{1}(\Pi(E))$, we define the map

$$
\bar{S}: \pi^{*} T B \longrightarrow \operatorname{pr}_{1}^{*}\left(T\left(Q_{1}(\Pi(E))\right)\right)
$$

with $\operatorname{pr}_{1}: \pi_{1}^{*}(E) \rightarrow Q_{1} \equiv Q_{1}(\Pi(E))$ and

$$
\bar{S}(e, v)=(((S \circ \pi)(e), e), T S(v)) .
$$

Also, we denote by

$$
\bar{P}_{2}: T Q_{1} \times_{T B} T E=T\left(\pi_{1}^{*} E\right) \longrightarrow T E
$$

the natural projection to the second factor.

With the previous notations, we are in a position to state the universal property (4.13) for linear connections. Namely, we have the following theorem. 
THEOREM 5.9. Let $(E, \pi, B)$ be a vector bundle with connected base and $Q_{1}(\Pi(E))$ be the connection bundle of the frame groupoid $\Pi(E)$. Then, the linear connection (5.23) of $\pi_{1}^{*} E$ satisfies the universal property

$$
C^{S}=\bar{P}_{2} \circ C^{\Gamma} \circ \bar{S}
$$

for any linear connection $c^{S}$ of $E$, corresponding to a section $S$ of $Q_{1}(\Pi(E))$.

Proof. The section $S$ corresponds to the connection $\gamma^{S}$ of $\Pi(E)$, which induces on $E$ the linear connection

$$
c^{S}: E \times_{B} T B \longrightarrow T E:(e, v) \longmapsto T_{\mathrm{id}} \overline{\mathrm{ev}}_{e}\left(\gamma^{S}(v)\right)
$$

(see Proposition 3.5 and Lemma 5.7). As in the proof of Proposition 3.5, $S(x)=$ $j_{x}^{1} s$ with $x=\pi(e)$. Thus, we get $\gamma^{S}(v)=T_{x} s(v)$ and

$$
c^{S}(e, v)=T_{\mathrm{id}} \overline{\mathrm{ev}}_{e}\left(T_{x} s(v)\right), \quad(e, v) \in E \times_{B} T B .
$$

Therefore, we check that

$$
\begin{aligned}
\left(\bar{P}_{2} \circ C^{\Gamma} \circ \bar{S}\right)(e, v) & =\left(\bar{P}_{2} \circ C^{\Gamma}\right)((S(x), e), T S(v)) \\
& =\bar{P}_{2}\left(T S(v), T_{\mathrm{id}} \overline{\operatorname{ev}}_{e}\left(T_{x} S\left(T_{S(x)} \pi_{1}\left(T_{x} S(v)\right)\right)\right)\right) \\
& =\bar{P}_{2}\left(T S(v), T_{\mathrm{id}} \overline{\operatorname{ev}}_{e}\left(T_{x} S(v)\right)\right) \\
& =T_{\mathrm{id}_{\mathrm{d}}} \overline{\mathrm{ev}}_{e}\left(T_{x} S(v)\right) \\
& =c^{S}(e, v),
\end{aligned}
$$

by which we conclude the proof.

For the sake of completeness, we note that, since $C^{\Gamma}$ is fully determined by the mapping $\bar{P}_{2} \circ C^{\Gamma}=: \hat{c}$, then the universal property (5.31) can be also written under the form

$$
c^{S}=\bar{S}^{*} \hat{c}
$$

which is analogous to the pullback expression of the universal connection of ordinary bundles.

Scholium 5.10. Under an appropriate interplay between $\pi_{1}^{*} E$ and the ordinary vector bundle of jets $J^{1} E$ of a vector bundle $(E, \pi, B)$ (the details of which are beyond the framework of this paper, dealing principally with groupoids), we can show (see [14]) that the universal connections of Section 5.2 are related with the universal connections on frame bundles considered in [3].

Acknowledgments. The authors express their sincere thanks to Professor K. Mackenzie for his comments and remarks on an earlier version of this paper, as well as to the referees for their suggestions. The present research was partially supported by University of Athens Special Research Grant 70/4/3410. 


\section{REFERENCES}

[1] R. Almeida and A. Kumpera, Structure produit dans la catégorie des algèbrö̈des de Lie [Product structure in the category of Lie algebroids ], An. Acad. Brasil. Ciênc. 53 (1981), no. 2, 247-250 (French).

[2] R. Brown, From groups to groupoids: a brief survey, Bull. London Math. Soc. 19 (1987), no. 2, 113-134.

[3] L. A. Cordero, C. T. J. Dodson, and M. de León, Differential Geometry of Frame Bundles, Mathematics and Its Applications, vol. 47, Kluwer Academic Publishers, Dordrecht, 1989.

[4] P. L. García, Connections and 1-jet fiber bundles, Rend. Sem. Mat. Univ. Padova 47 (1972), 227-242.

[5] P. J. Higgins and K. Mackenzie, Algebraic constructions in the category of Lie algebroids, J. Algebra 129 (1990), no. 1, 194-230.

[6] S. Kobayashi and K. Nomizu, Foundations of Differential Geometry, Vol. I, Interscience Publishers, New York, 1969.

[7] _ Foundations of Differential Geometry. Vol. II, Interscience Tracts in Pure and Applied Mathematics, no. 15, Interscience Publishers, New York, 1969.

[8] S. Kumar, A remark on universal connections, Math. Ann. 260 (1982), no. 4, 453462.

[9] K. Mackenzie, Lie Groupoids and Lie Algebroids in Differential Geometry, London Mathematical Society Lecture Note Series, vol. 124, Cambridge University Press, Cambridge, 1987.

[10]__ Lie algebroids and Lie pseudoalgebras, Bull. London Math. Soc. 27 (1995), no. 2, 97-147.

[11] Nagô van Quê, Du prolongement des espaces fibrés et des structures infinitésimales, Ann. Inst. Fourier (Grenoble) 17 (1967), no. 1, 157-223 (French).

[12] M. S. Narasimhan and S. Ramanan, Existence of universal connections, Amer. J. Math. 83 (1961), 563-572.

[13] _ Existence of universal connections. II, Amer. J. Math. 85 (1963), 223-231.

[14] A. Nikolopoulos, On universal vector bundle connections, manuscript.

[15] J. Pradines, Théorie de Lie pour les groupoïdes différentiables. Calcul différentiel dans la catégorie des groupoïdes infinitésimaux, C. R. Acad. Sci. Paris Sér. A-B 264 (1967), A245-A248 (French).

[16] E. Vassiliou, Connections on 1-jets principal fiber bundles, Rend. Sem. Mat. Univ. Padova 56 (1976), 23-31.

[17] - On the canonical connection of the 1-jet bundle of a principal bundle, Bull. Soc. Roy. Sci. Liège 47 (1978), no. 1-2, 5-11.

[18] A. Weinstein, Symplectic groupoids and Poisson manifolds, Bull. Amer. Math. Soc. (N.S.) 16 (1987), no. 1, 101-104.

Efstathios Vassiliou: Department of Mathematics, University of Athens, Panepistimiopolis, Athens 15784, Greece

E-mail address: evassi1@cc.uoa.gr

Apostolos Nikolopoulos: Department of Mathematics, University of Athens, Panepistimiopolis, Athens 15784, Greece

E-mail address: ni kolopou1os@synigoros.gr 


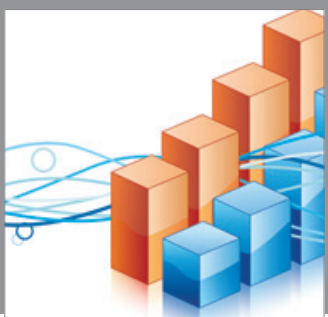

Advances in

Operations Research

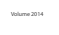

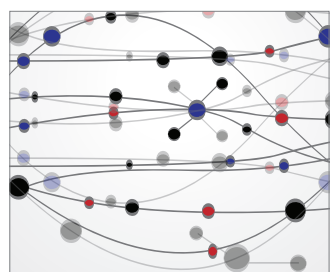

\section{The Scientific} World Journal
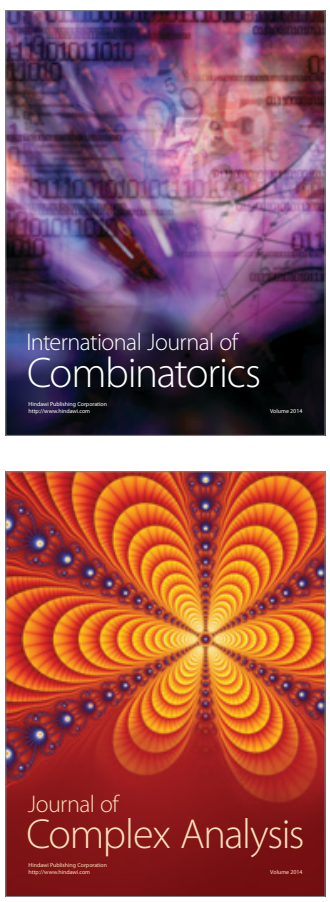

International Journal of

Mathematics and

Mathematical

Sciences
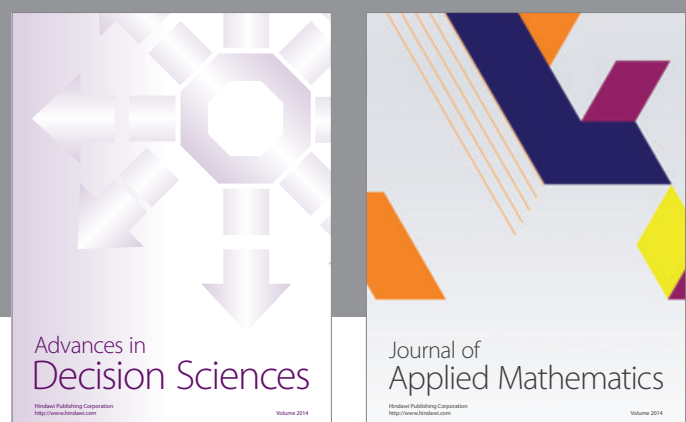

Journal of

Applied Mathematics
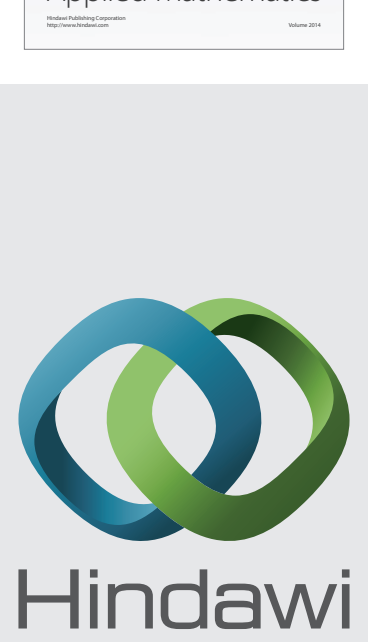

Submit your manuscripts at http://www.hindawi.com
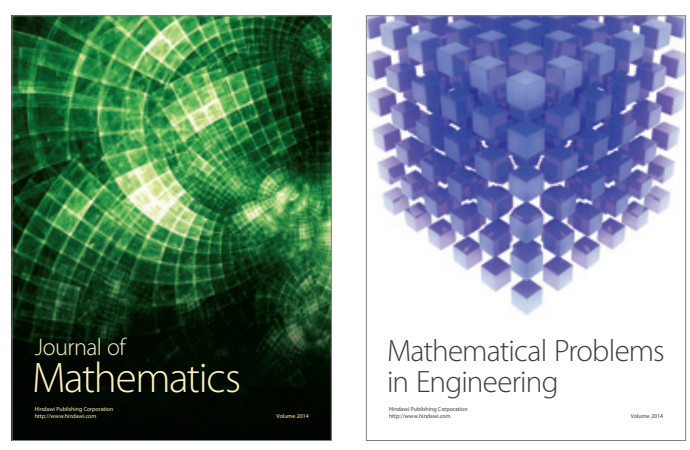

Mathematical Problems in Engineering
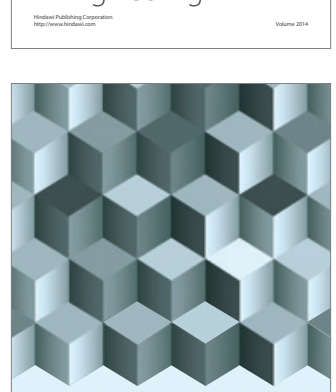

Journal of

Function Spaces
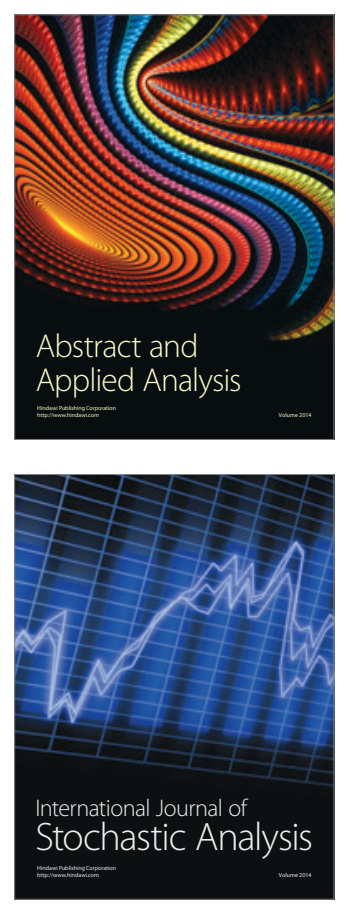

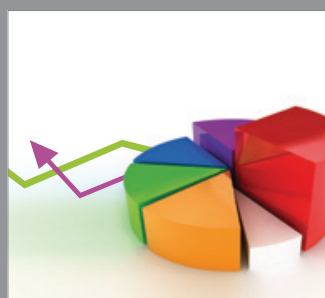

ournal of

Probability and Statistics

Promensencen
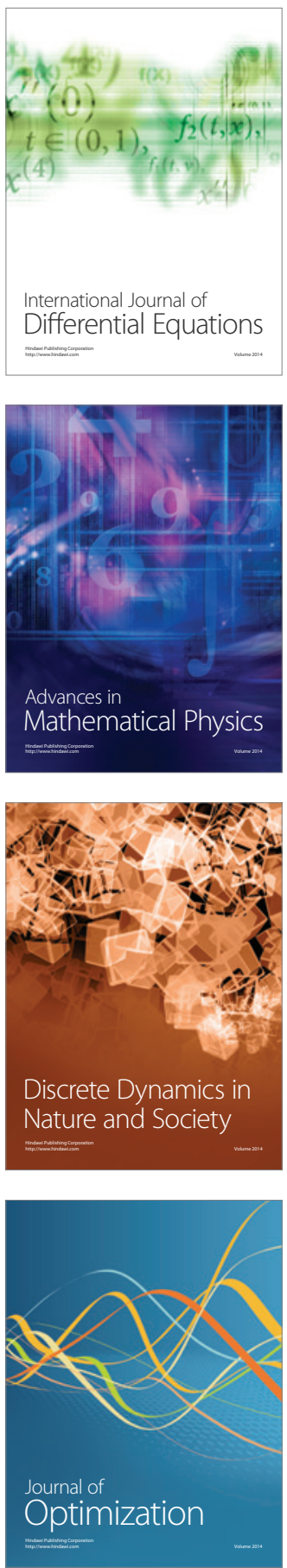